\title{
Diagnostic Dilemma in a Carotid Body Tumor: A Case Report
}

\author{
Islam $\mathrm{A}^{1 *}$, Sabah $\mathrm{N}^{2}$, Chowdhury $\mathrm{NH}^{1}$, Mohammad TT ${ }^{1}$ and Aga $\mathrm{S}^{2}$ \\ ${ }^{1}$ Department of ENT \& HNS, Bangladesh medical college, Bangladesh \\ ${ }^{2}$ National Institute of Cardiovascular Diseases, Bangladesh
}

*Corresponding author: Md Ashraful Islam, Department of ENT and Head \& Neck surgery, Bangladesh Medical college Hospital, Dhaka, Bangladesh, Email: Ashrafis123@yahoo.com

\section{Case Report}

Volume 6 Issue 2

Received Date: June 20, 2021

Published Date: July 05, 2021

DOI: $10.23880 /$ ooaj-16000214

\section{Abstract}

An asymptomatic swelling in the neck region has many differential diagnoses. Most common of them is reactive lymphadenitis and carotid body tumor being the last thing. We present here a case of carotid body tumor that misguided us on clinical and radiological ground. It was suspected intra-operatively and confirmed by histopathological examination. Carotid body tumors are rare neoplasm. It arises from the chemoreceptor cells of the carotid bulb. We report a case of carotid body tumor in a 57-year-old female, who presented with painless, gradually progressive lateral neck swelling. The diagnosis is suspected on the basis of clinical radiological and per-operative examination findings. Histopathological examination confirms the diagnosis of carotid body tumor.

Keywords: Carotid Body Tumor; Neck Swelling; Paragangliomas

Abbreviations: PGLs: Paragangliomas; CBT: Carotid Body Tumor; CT: Computed Tomography; MRI: Magnetic Resonance Imaging; DSA: Digital Subtraction Angiography; PRRT: Peptide Receptor Radionuclide Therapy.

\section{Introduction}

Paragangliomas (PGLs) are uncommon tumors with the incidence of about 1-2per 100,000. Only (3\%) of all PGLs occur within the head and neck and the majority originates in the carotid body [1]. Carotid body tumor (CBT) is the most frequently seen paragangliomas of head and neck, arising from the carotid body chemoreceptors but rarely seen clinically. As it is rarely seen, its clinical diagnosis and management is a challenge for the clinician. Most patients remain unaware about this lesion as it remains asymptomatic. Most of them take medical treatment by the quack for the neck swelling and some comes to general physician with the complain of slow growing neck mass and pain [2]. Here we are going to discuss the diagnostic dilemma we faced in a case of carotid body tumor.

\section{Case Report}

A 57-year-old female presented with a slow growing right sided neck swelling of 10 years duration. She had a feeling of discomfort in the region on and off but had no other symptom. On examination, there was a single swelling below the angle of the mandible, firm, non-tender, non-pulsatile, mobile, free from the skin and underlying structure (Figure 1).

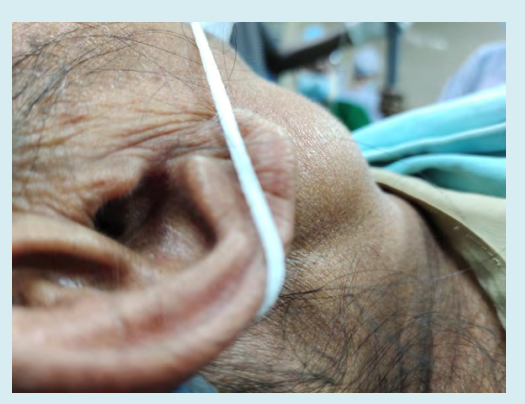

Figure 1: Pre -operative image showing swelling in the retromandibular region. 
On intra-oral examination there was no abnormality. No swelling on floor of mouth. Clinically it was diagnosed as para pharyngeal mass. Our differential diagnosis was Cervical lympahdenopathy, Branchial cyst, Shwannoma, Neurofibroma, Paraganglioma.

Patient brought to us with an FNAC that was suggestive of reactive hyperplasia. MRI of neck revealed large isointense mass in carotid triangle ( $47 \times 51 \mathrm{~mm}$ ) in between internal and external carotid (Figure 2). On T2 it was hyperintense with multiple flow voids (Figures $3 \& 4$ ). MR Angiogram done to see vascularity of the mass and to detect feeding vessels. There was positive Lyre's sign (Splaying of carotid arteries), but no significant vascularity within the lesion (Figure 5).

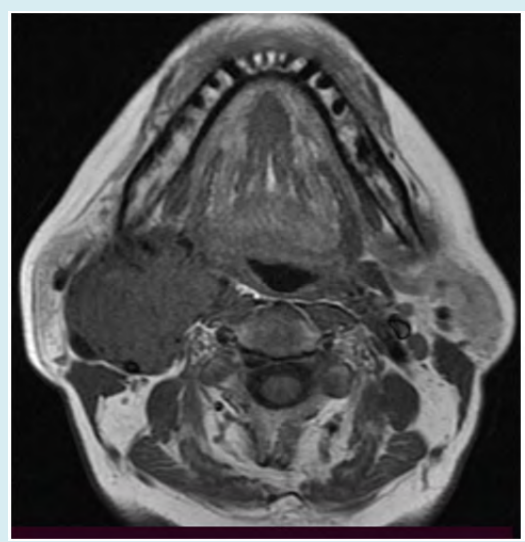

Figure 2: T1 MRI showing isointense lesion.

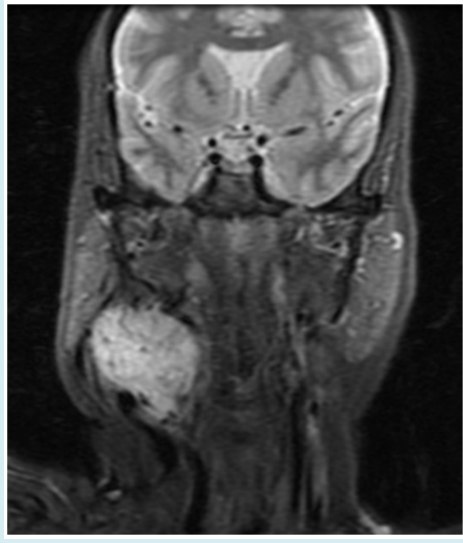

Figure 3: T2 MRI showing hyperintense lesion with multiple flow voids.

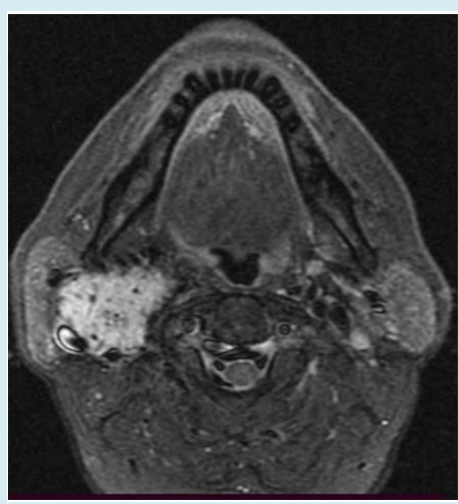

Figure 4: STAIR image showing hyperintense lesion with multiple flow voids.
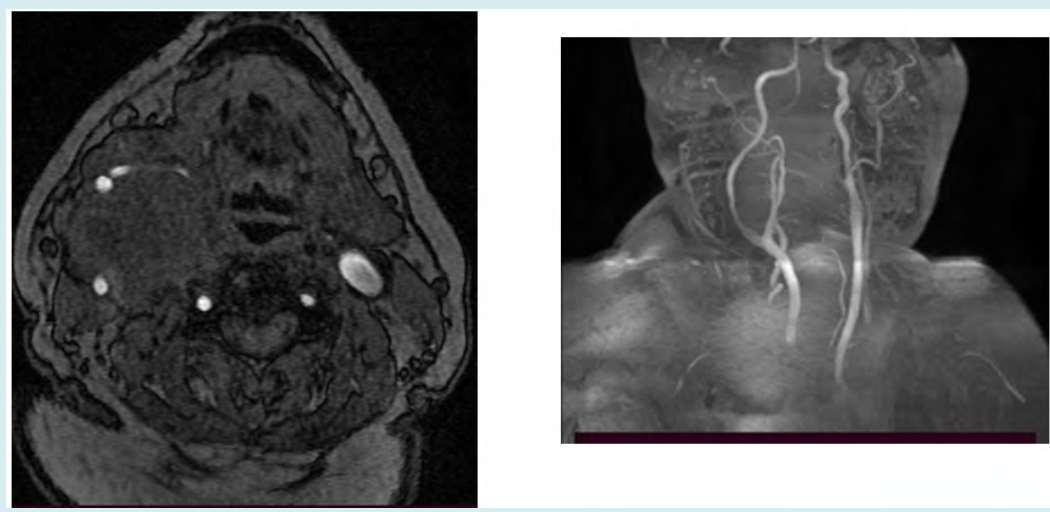

Figure 5: MRA showing splaying of carotid vessels by the tumor, there is no enhancement of mass by the contrast.

As clinically it was non pulsatile, no history of faint, FNAC suggested the lesion as reactive hyperplasia, MRA did not showed vascularity though on T2 MRI there was flow voids, the lesion was supposed to us as a Schwannoma (Vagal). On clinical and radiological basis, we planned for excision of the lesion. We kept one senior vascular surgeon in our team as the lesion showed multiple flow voids on T2, with the suspension of CBT. Patient was prepared appropriately and 
procedure was performed under General anesthesia.

A transverse skin incision was given on the side of tumor, subplatysmal flaps were raised both upper and lower, sternocleidomastoid retracted and mass was delineated (Figures $5 \mathrm{~A}-\mathrm{E}$ ). There was a $2^{\mathrm{x}} 1.5 \mathrm{~cm}$ sized lymph node over the carotid bifurcation. That was excised. Intra-operatively mass was seen arising between right external and internal carotid vessels. Location of the tumor was suggestive of carotid body tumor. It was very meticulously excised from the walls of both arteries with minimal blood loss, Small arterial branches arising from the bifurcation had to be cauterized. The postoperative recovery was uneventful. Specimen sent for histopathological examination, which confirmed the diagnosis of carotid paraganglioma.

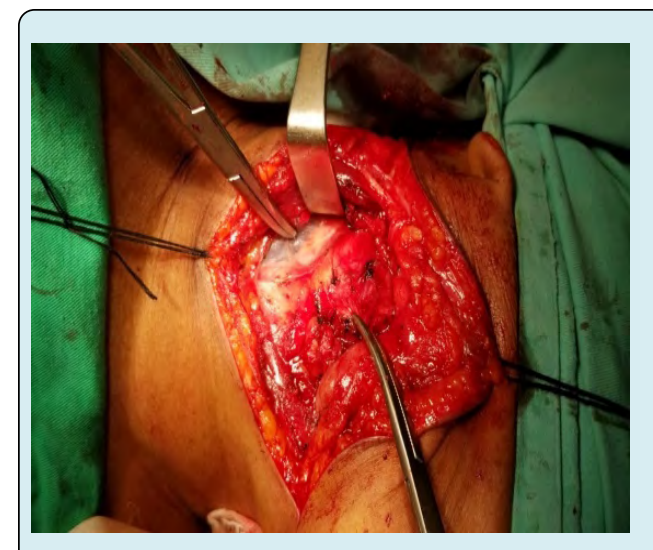

A

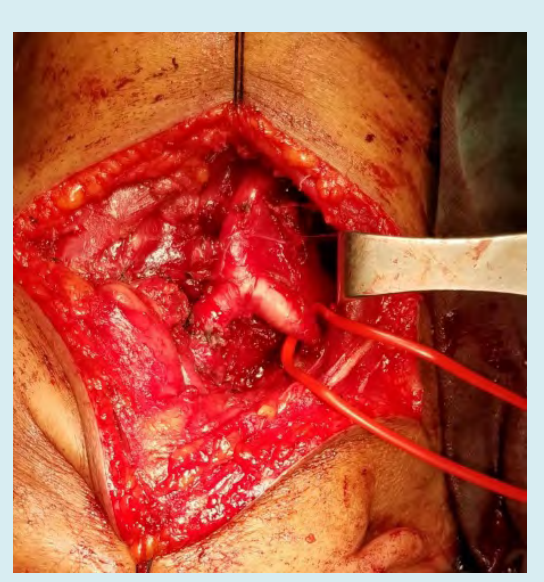

B

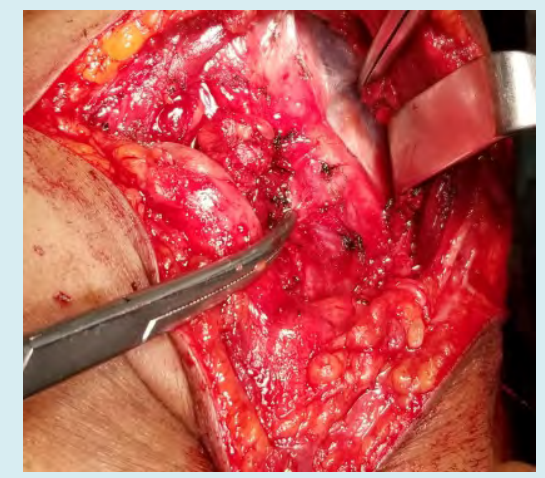

C

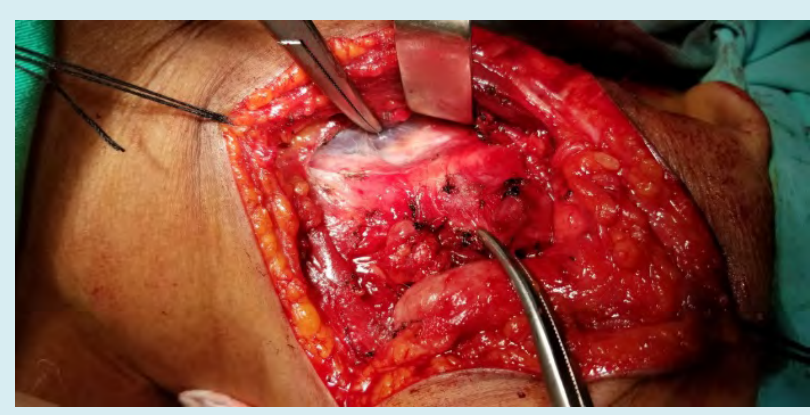

$\mathrm{D}$

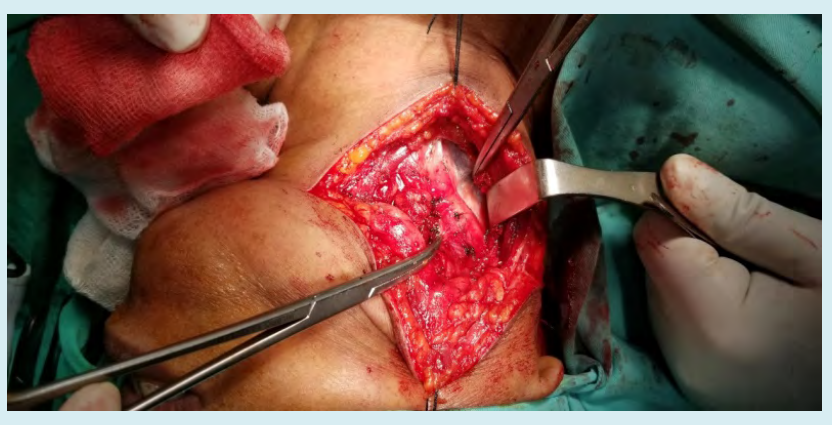

$\mathrm{E}$

Figures 5A-E: Sequential image showing dissection of the lesion. (E) Showing bifurcation of Carotid after resection of the tumor.

\section{Discussion}

Carotid body is a reddish brown structure arises from tunica adventitia of the common carotid artery. Von Haller in 1743 was the first described it. Afferent innervation is provided by the glossopharyngeal nerve. The carotid body detects changes in the composition of arterial blood and initiates an autonomic reflex inresponse to hypercapnia, hypoxia. Hypoxia and genetic factors are considered to be involved in formation of carotid body tumors, even though the exact pathogenesis remains unknown. It is often enlarged in people living at higher altitudes.

Carotid body tumors (CBTs) also known as paragangliomas or chemodectomas that are derived from the embryonic neural crest accounts for $0.6 \%$ of the head and neck tumors in humans. These are rare neuroendocrine neoplasms which arise near the carotid bifurcation within glomus cells. Feeding vessels run primarily from the ascending pharyngeal branch of the external carotid artery and innervated through the glossopharyngeal nerve. It is attached to the posteromedial wall of the vessel at its 


\section{Otolaryngology Open Access Journal}

bifurcation.

The majority of these tumors are asymptomatic initially. The most observed symptoms are pain and swelling. Dysphagia, and autonomic dysfunction may occurs in symptomatic cases $[3,4]$. Commonly seen at the age of 50 to 70 years with higher incidence in female [2]. Pressure symptoms mostly seen due to pressure over the hypoglossal nerve, glossopharyngeal nerve, vagus nerve and sympathetic chain [5]. Clinical history, examination, and radiological diagnosis are the keystones to diagnosis and management. Ultrasound, Computed tomography (CT) and Magnetic resonance imaging (MRI) are useful radiological tools in diagnosis. Yet Angiography is essential to study about the vascular anatomy $[1,2,6]$. Color Doppler sonography and digital subtraction angiography (DSA) play a vital role in the confirmation of the clinical diagnosis of CBTs and DSA is regarded as the gold standard for the final diagnosis of CBTs. Subtraction computed tomographic angiography can facilitate the 3D-reconstructed image that can help demonstrate more directly the relationship of the tumor with the surrounding tissues [5]. Angiographic methods allow the evaluation of the vessels supplying the tumor and preoperative embolization [2].

Surgical excision still remains the gold standard therapeutic modality for the treatment of CBTs. Radiotherapy is an alternative treatment modality which may decrease the tumor size or stop its growth. It is recommended for patients who cannot undergo surgery on account of extensive involvement, multiple tumors, and high operative and anesthetic risk [2]. Peptide receptor radionuclide therapy (PRRT) can be a therapeutic option in selected cases of head and neck paragangliomas, including tumors that cannot be removed, occult, recurrent, malignant, or metastatic lesions [6].

The surgical resection of carotid PG poses a risk of immediate and late complications. However, the rate of surgical complications depends on size of tumor and expertisation of surgeons. Permanent nerve palsy and vascular complications are described in $2.3 \%$ of type I/II tumors and in $35.7 \%$ of type III tumors. Surgical procedure may also be complicated by profuse bleeding due to the high vascularity of the tumor. The malignant potential of CBTs has been estimated at between 5 and 10\%, but in young patients, the rate is higher [1,2].

In our patient clinically there was no pulsation over the tumor, there was no history of faintness. The location of the lesion was on retromandibular region. Patient also came to us with a FNAC that was suggestive of reactive hyperplasia. MRI showed a 4 × $3 \mathrm{~cm}$ lobulated well-defined lesion at the bifurcation of carotid that was isointense on
$\mathrm{T} 1$ and hyperintense on $\mathrm{T} 2$ with multiple flow voids. On MR angiography there was splaying of the external and internal carotid artery but no significant enhancement. All above clinical and radiological finding was not conclusive to diagnose a CBT. There are multiple literatures on google search that shows atypical presentation of CBTs $[7,8]$.

In our case during surgery we noted a lymph node just over the bifurcation from where FNAC might be taken that confused us. There are few literatures where they face similar problem due to FNAC from a lymph node $[7,8]$. Though FNAC is contraindicated in a suspected case of CBT, in our case the patient came to us with FNAC report suggested by other physician. In our case we also had been confused due to non-enhancement of tumor on MRA. Complete surgical excision was done. Shamblin, et al. [8] classified the tumor in to 3 grades. Grade I describes localized mass where tumor don not involve more than $180^{\circ}$ of carotid artery, grade II includes where the tumor involves more than $180^{\circ}$ and grade II includes complete encasement of carotid artery by the tumor in our case it was Shamblin grade II. Our diagnosis was confirmed by histopathology.

\section{Conclusion}

A high index of suspicion is necessary to diagnose a case of carotid body tumor. Imaging is essential; especially MRI and MRA. DSA is gold standard. Once suspected, FNAC should be avoided. In a upper lateral neck swelling though rare, CBT should always be in differential diagnosis. In such cases at least an color Doppler ultrasonography should be the investigation of choice before doing FNAC. During surgery in such confusing cases a vascular surgeon should always be included in the surgical team.

\section{References}

1. Hoang VT, Trinh CT, Lai TAK, Doan DT, Tran TTT (2019) Carotid body tumor: a case report and literature review. J Radiol Case Rep 13(8): 19-30.

2. Arora N, JaiLal D, Jyoti S (2016) Carotid Body Tumor with Unusual Presentation: a case report. International Journal of Community Health and Medical Research 2(1): 24-26.

3. Taha AY (2015) Carotid Body Tumours a Review. IJCM 6(3): 119-131.

4. Kachewar SG, Sankaye SB (2012) Carotid body tumor presenting as an incidentaloma. JMGIMS 17(2): 64-66.

5. Ma D, Liu M, Yang H, Ma X, Zhang C (2010) Diagnosis and surgical treatment of carotid body tumor. A report of 18 cases. JCDR 1(3): 122-124. 
6. Gupta SK, Singla S, Karunanithi S, Damle N, Bal C (2014) Peptide receptor radionuclide therapy with (177)Lu DOTATATE in a case of recurrent carotid body paraganglioma with spinal metastases. Clin Nucl Med 39(5): 440-441.

7. Chand P, Singh R, Singh B, Singla RL (2015) Carotid Body Tumor Presenting as Parotid Swelling Misdiagnosed as
Pleomorphic Adenoma: A Rare Presentation. NJS 21(2): 157-158.

8. Shamblin WR, Remine WH, Sheps SG, Harrison EG Jr (1971) Carotid body tumor (chemodectoma): clinicopathologic analysis of ninety case. Am J Surg 122(6): 732-739. 